\title{
Denavit-Hartenberg Coordinate System for Robots with Tree-like Kinematic Structure
}

\author{
Aleksander K. Kovalchuk, Faniya Kh. Akhmetova \\ Bauman Moscow State Technical University, Russia
}

\begin{tabular}{l}
\hline \hline Article Info \\
\hline Article history: \\
Received Aug 13, 2016 \\
Revised Oct 3, 2016 \\
Accepted Nov 16, 2016 \\
\hline
\end{tabular}

\section{Keyword:}

Tree-like actuating mechanism Denavit-Hartenberg coordinate system

Robot kinematics

Robotic actuating mechanism

Robot dynamics

\begin{abstract}
The paper presents a modified Denavit-Hartenberg coordinate system resulted from joint application of graph theory and the Denavit-Hartenberg coordinate system, which was developed to describe the kinematics of robot actuators with a linear open kinematic chain. It allows forming mathematical models of actuating mechanisms for the robots with tree-like kinematic structures. The work introduces the concept of primary and auxiliary coordinate systems. It considers an example of making the links' reachability matrix and reachability graph for the tree-like actuating mechanism of a robotic mannequin. The use efficiency of the proposed modified DenavitHartenberg coordinate system is illustrated by the examples giving the mathematical description of the kinematics and dynamics of specific robots' tree-like actuating mechanisms discussed in the previously published papers. It is shown that the proposed coordinate system can also be successfully applied to describe the actuating mechanisms of robots with a linear open kinematic chain, which is a particular case of the tree-like kinematic structure. The absence of branching joints in it does not require introducing auxiliary coordinate systems and the parameters $f(i)$ and $n s(i)$ are necessary only for the formal notation of equations, which have similar forms for the tree-like and linear chains. In this case, the modified and traditional coordinate systems coincide.
\end{abstract}

Copyright (C) 2016 Institute of Advanced Engineering and Science. All rights reserved.

\section{Corresponding Author:}

Aleksander K. Kovalchuk,

Bauman Moscow State Technical University,

5/1, 2nd Bauman Str., Moscow, Russia, 105005.

Email: editor@academicpapers.org

\section{INTRODUCTION}

The methods of mathematical description of the robots' actuator kinematics and dynamics are extensively described in literature [1-6]. The authors consider an actuating mechanism (AM) as a linear open kinematic chain; that is typical for industrial robots and special-purpose handling equipment. There are two most widely used methods to describe the kinematics and dynamics of such robots' AMs.

The first method is based on the use of block matrices [7, 8]. It allows obtaining kinematics equations in both analytic and algorithmic forms. To use this method in practice, the authors developed software that allows exploring and designing robotic actuating systems, including those with flexible links. The work of A. G. Leskov et al. [9] has an example of using this method to build a model of the actuator kinematics in an industrial robot with a branching kinematic structure (KS) of gripping.

The second method to describe the robotic actuator kinematics was offered by J. Denavit and R. S. Hartenberg [10]. It is based on using homogeneous transformation matrices $(4 \times 4)$ giving unambiguous and clear rules for making a robot's actuator mathematical model. The number of parameters in the matrix $A_{i}$ of the actuator serial links' relative position is minimal and naturally defines the mutual arrangement of actuator serial links. The form of the matrix $A_{i}$ is similar for both rotational and translational joints. 
The significant advantage of this method developed to construct related coordinate systems (CS) is that you may specify only four parameters determining the relative position of two serial CSs $i-l$ and $i$ and, as a consequence, the transformation matrix $A_{i}$.

The resulting matrix $A_{i}$ joining the CSs $i-1$ and $i$ has the following form [10]:

$$
A_{i}=\left|\begin{array}{cccc}
\cos \left(\theta_{i}\right) & -\cos \left(\alpha_{i}\right) \cdot \sin \left(\theta_{i}\right) & \sin \left(\alpha_{i}\right) \cdot \sin \left(\theta_{i}\right) & a_{i} \cdot \cos \left(\theta_{i}\right) \\
\sin \left(\theta_{i}\right) & \cos \left(\alpha_{i}\right) \cdot \cos \left(\theta_{i}\right) & -\sin \left(\alpha_{i}\right) \cdot \cos \left(\theta_{i}\right) & a_{i} \cdot \sin \left(\theta_{i}\right) \\
0 & \sin \left(\alpha_{i}\right) & \cos \left(\alpha_{i}\right) & d_{i} \\
0 & 0 & 0 & 1
\end{array}\right|
$$

Out of the four parameters $\left(\theta_{i}, d_{i}, a_{i}, \alpha_{i}\right)$ from expression (1), the two parameters $a_{i}$ and $\alpha_{i}$ are always constant and determined by the robot's actuating mechanism design. One of the other two parameters $\left(\theta_{i}\right.$ or $\left.d_{i}\right)$ is variable. For a rotational joint, the value $\theta_{i}$ specifies the angle of relative rotation of the links $i-1$ and $I$, the linear value $d_{i}$ is constant. For a telescopic joint it is vice versa, the variable value is $d_{i}$. The variable value of the $i$-th joint $\left(\theta_{i}\right.$ or $\left.d_{i}\right)$ is commonly referred to as the generalized coordinate of the robot's actuating mechanism.

In building robotic kinematic models, this method is most prevalent among developers due to its clarity and reference to actuators' design parameters.

However, the attempts of using this method of describing the robots with a tree-like KS revealed certain difficulties in its application.

\section{APPLICABILITY}

During the process of creating walking robots (WR) extensively elaborated lately, the developers came across a problem - how to mathematically describe the kinematics and dynamics of their actuating mechanisms, which are spatial tree-like kinematic structures with a large number of freedom motion degrees. Well-known methods providing good results in the description of robots' AMs with linear open kinematic chains are not always acceptable to describe the AMs with tree-like kinematic chains.

It is also important that the mathematical models of actuator kinematics obtained by these methods are to be used in constructing dynamical equations and algorithms for robot motion control.

Therefore, the development of new effective methods to construct the mathematical models of robotic tree-like actuator kinematics and dynamics is an important scientific and technical challenge.

\section{RESEARCH METHOD}

The authors suggest a method of building a modified Denavit-Hartenberg (DH) coordinate system; it allows forming mathematical models for the actuators of robots with arbitrary tree-like KSes. This method is based on joint application of the graph theory [11] and DH coordinate system proposed by Denavit and Hartenberg [10] to describe the kinematics of robots with linear open kinematic chain. This saves the known benefits of the DH CS and gives a possibility to describe the kinematics of the arbitrary tree-like KS using the graph theory methods.

\section{RESULTS AND ANALYSIS}

Let us consider a kinematics scheme of a robot's actuating mechanism (Fig. 1) in a tree-like directed graph (Fig. 2) [11]. In this graph, the actuating links are vertices and the connecting joints are edges [12]. Let us assume that the tree root (the link number 0) is the robot's surrounding space. The robotic actuating links start from number 1; then their numbers increase from the root of the tree to its leaves without gaps. There must be a condition that the link's own number is smaller than the number of its any link-successor. The number of the AM's generalized coordinate (as well as the number of the corresponding joint) is the same as the number of the link connected by this joint to the previous link. For walking robots, the body of which is not attached to a fixed base, the total number of its freedom degrees is equal to $N+6$, where $N$ is the number of motion freedom degrees for its actuating mechanisms. To "bind" the WR's AMs to an absolute CS and describe its motion in space, we introduce a dummy kinematic chain that connects the robot's body with a stationary (in the absolute CS) dummy stand. This dummy chain consists of weightless links $(0 \div 5)$, (three translational and three rotational kinematic pairs, 5th grade) and characterizes the position and orientation of the robot's body in the absolute CS.

Such a description of the robot's tree-like actuating mechanism kinematic scheme presumes different variants of the links' numbering. The quantity of numbering variants depends on the tree-like kinematic structure's complexity. Here, every numbering variant corresponds to its own directed graph, its

Denavit-Hartenberg Coordinate System for Robots with Tree-like Kinematic Structure (Aleksander K) 
reachability matrix and its own block vector $\bar{Z}$ determining the sequence of basis vectors of the axes $\bar{Z}$, which match the joints connecting the actuating links with their ancestor links.

A developer is given the right to choose a specific numbering variant based on his priorities. However, we must remember that all numbering options are equivalent and lead to the desired result.

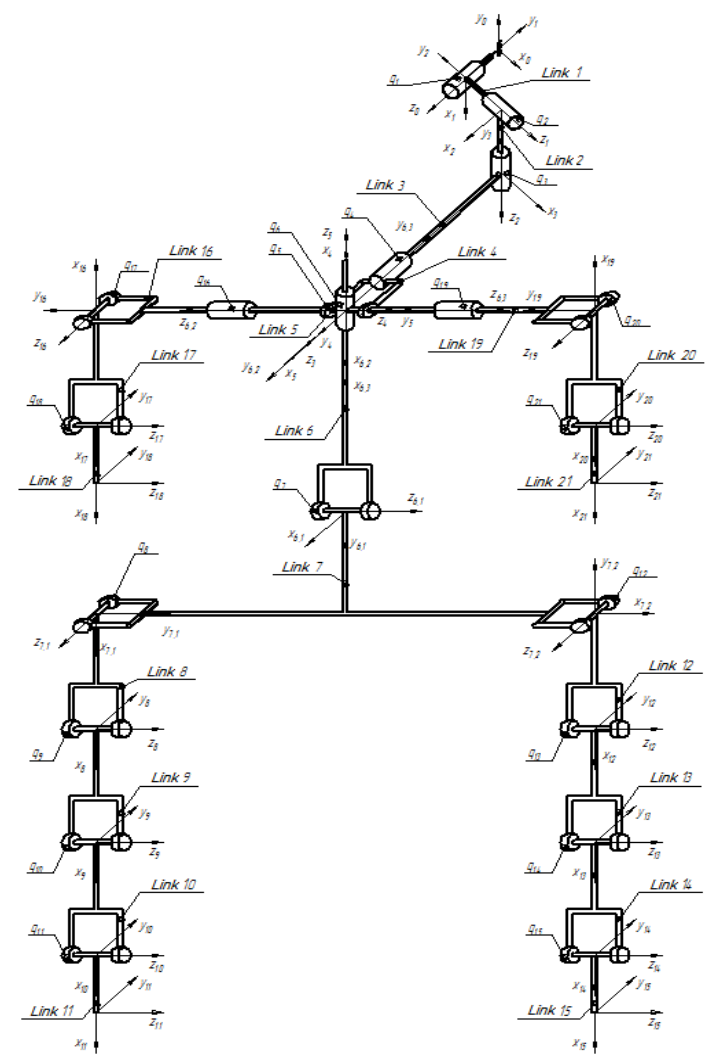

Figure 1. Kinematic scheme of a robotic mannequin's actuating mechanism with dummy links

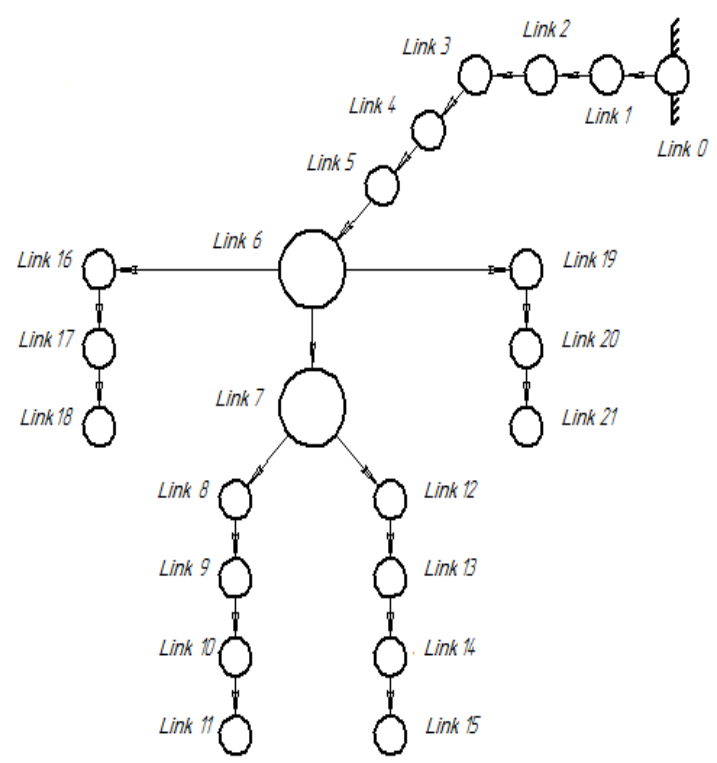

Figure 2. Tree-like graph representing the kinematic structure of a robotic mannequin's actuating mechanism 
In the mathematical description of the kinematic structure of robotic AMs represented in the form of tree-like directed graphs, we use the following definitions [12]:

$L=\{1,2 \ldots, N\}-$ the irregular set, the elements of which are the numbers of AM's links;

$f(i)$ - the number of ancestor of the link $i$;

$s(i, k)$ - the number of the link - $k$-th successor of the link $i$;

$d g^{+}(i)$ - the outdegree of the link $i$; it determines the number of successors of the link $i$;

$\Gamma(i) \quad-$ the sequence of links' numbers, which are the successors of the link $i ; \Gamma(\mathrm{i})=\{(\mathrm{s}(i, 1)$,

$\left.\left.\mathrm{s}(i, 2), \ldots \mathrm{s}(i, \mathrm{k}), \ldots \mathrm{s}\left(i, d g^{+}(i)\right)\right)\right\}$;

$n s(i)$ determines the order number of the successor link $i$ relatively its ancestor;

$\sigma_{i}=\{0,1\}$ - the coefficient to define the joint type of the link $i(1$ - rotational, 0 - translational);

$\sigma_{i}=\operatorname{diag}\left\{\sigma_{1}, \sigma_{2}, \ldots \sigma_{N}\right\}$ - the diagonal matrix defining the joint types of the tree-like AM' links.

Let us consider the procedure on setting the coordinate systems related to the tree-like AM's links $[13,14]$. Every AM's link is connected with the quantity of coordinate systems equal to the quantity of its successors. One CS connected with the link is regarded as primary, other CSes are auxiliary. Fig. 3 shows three sample coordinate systems connected with the link $\boldsymbol{i}$, which has three successors. All CSes are assigned in accordance with Denavit-Hartenberg [10] rules.

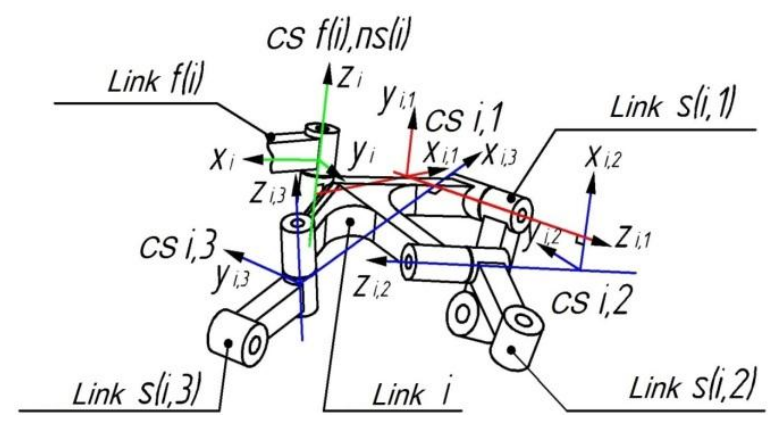

Figure 3. Designation of coordinate systems connected with a branching link

The transition of the link $i$ from the primary CS to the corresponding CS of its ancestor link $f(i)$ is defined by the transformation matrix $A_{i}$ :

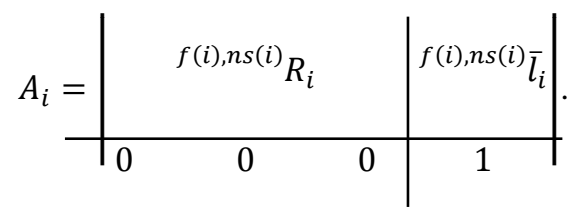

The transitions of the link $i$ from auxiliary CSes to the primary CS is determined by the constant matrices of homogeneous transformations $M_{i, n s(j)}$, where $j$ is the number of the $i$-link's successors:

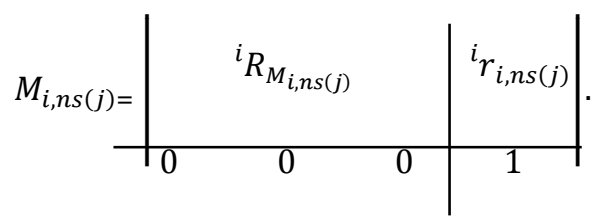

The expressions (2) and (3) allow writing recurrent relations to determine the matrix $T$, which specifies the transition from primary links' CSes to the absolute CS.

$$
T_{i}=T_{f(i)} M_{i, n s(j)} A_{i}
$$

The matrix $M_{i, n s(j)}$ is always constant and performs a supporting function during the transition from the successor's primary CS to the ancestor's primary CS. In case $j \in \Gamma_{(i)}$ and it corresponds to the primary CS of the link $i$, then $M_{i, n s(j)=} E$. 
Coordinate system sequence order in the tree-like kinematic structure of robots' AMs is set with the help of the block vector $\bar{Z}$. It defines the sequence of the basis vectors of the axes $z$, which correspond to the joints connecting the actuating links with their ancestors. For the actuating mechanism, the kinematic scheme of which is presented in Fig. 1, the block vector $\bar{Z}$ has the following form:

$$
\begin{aligned}
& \bar{Z}=\left({ }^{0} z_{1},{ }^{0} z_{2},{ }^{0} z_{3},{ }^{0} z_{4},{ }^{0} z_{5},{ }^{0} z_{6,1},{ }^{0} z_{7,1},{ }^{0} z_{8},{ }^{0} z_{9},{ }^{0} z_{10},{ }^{0} z_{11},{ }^{0} z_{7,2}\right. \text {, } \\
& \left.{ }^{0} z_{12},{ }^{0} z_{13},{ }^{0} z_{14},{ }^{0} z_{15},{ }^{0} z_{6,2},{ }^{0} z_{16},{ }^{0} z_{17},{ }^{0} z_{18},{ }^{0} z_{6,3},{ }^{0} z_{19},{ }^{0} z_{20},{ }^{0} z_{21}\right)^{T}
\end{aligned}
$$

The first element (it corresponds to the first link) is $z_{0}$ in the absolute CS. The second element is the basis vector of the first link's axis $z$. For instance, for Link 19, there is the basis vector of the axis $z$ of the sixth link's auxiliary CS. The axes of the links-leaves' coordinate systems are not included into this block vector.

It should be noted that in the considered example, the $\bar{Z}$ block vector's dimension is $(24 \times 24)$, and the $\bar{Z}$ block vector's dimension is $(21 \times 21)$ - due to the fact that the elements ${ }^{0} z_{6,2},{ }^{0} z_{6,3},{ }^{0} z_{7,2}$ of the vector $\bar{Z}$ are not the AM's kinematic pair axes but are related with the robot's auxiliary coordinate systems determining their position and orientation in the main coordinate system. For a linear open kinematic chain, the dimensions of the vectors $\bar{Z}$ and $\bar{Z}$ coincide.

For the mathematical description of the AM's tree-like kinematic structure, it is necessary to determine the links' order relative to each other. It is found by the reachability matrix $D$ - the square matrix, every element of which $d_{i j}=1$ if the $i$-th vertex of the directed graph describing the AM's kinematic structure is reachable from the vertex $j$ and $d_{i j}=0$ if the $i$-th vertex is not reachable from the vertex $j$.

Upon numbering the robot's actuating links in accordance with the above rules, the reachability matrix $D$ turns out to be a lower triangular matrix, its dimension is equal to the actuating links' number. Therefore, both the tree-like graph representing the robot's tree-like kinematic structure and the reachability matrix $D$ reflect the relative position and reachability of the actuating mechanism's links.

Thus, the proposed method of constructing a modified DH coordinate system allows defining the values of the tree-like actuating mechanism's primary and auxiliary coordinate systems. Tables 1 and 2 give the modified DH parameters' values for primary and auxiliary coordinate systems of the robotic actuating mechanism, the kinematic scheme of which is presented in Figure 1 [11].

Table 1. Modified DH parameters' values for primary coordinate systems of a robotic actuating mechanism

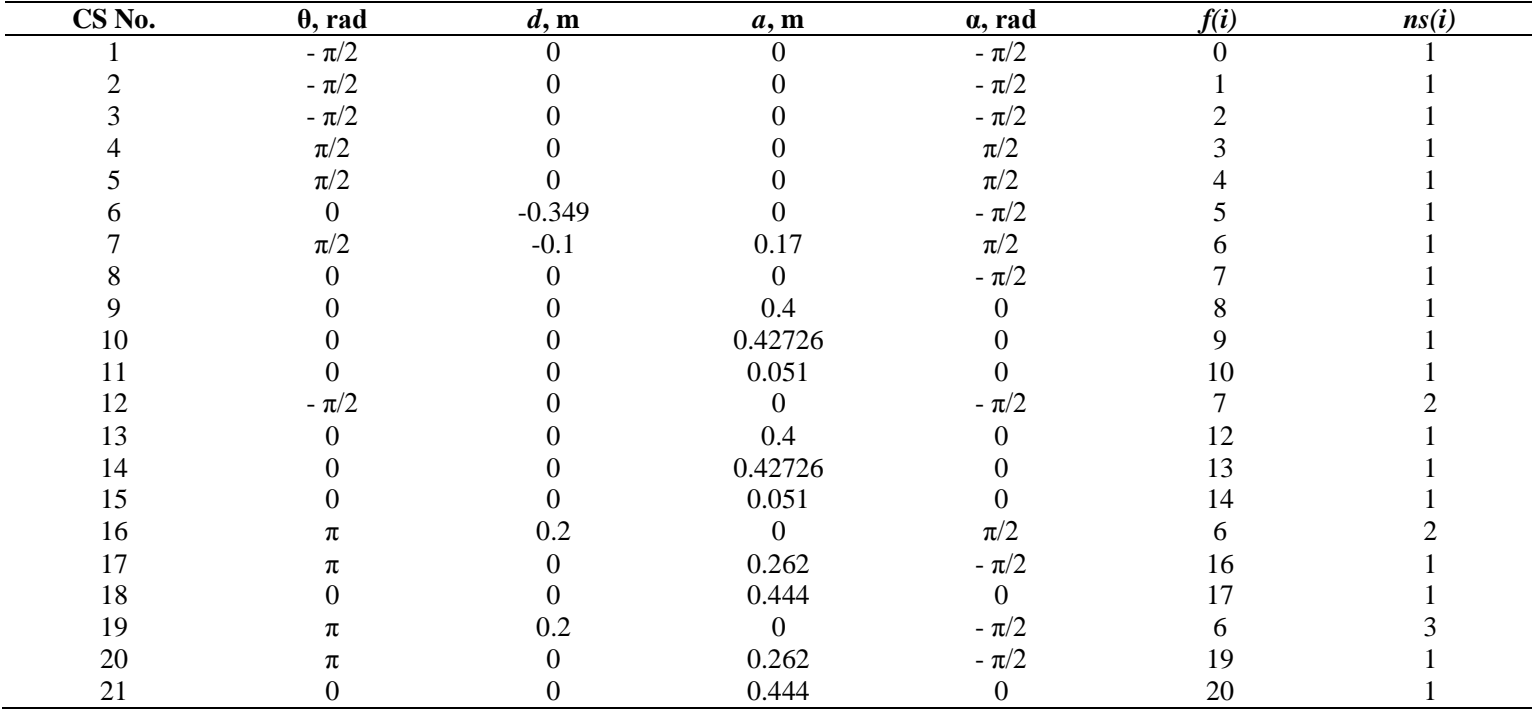

Table 2. Modified DH parameters' values for auxiliary coordinate systems of a robotic actuating mechanism

\begin{tabular}{ccccccc}
\hline CS No. & $\boldsymbol{\theta}, \mathbf{r a d}$ & $\boldsymbol{d}, \mathbf{m}$ & $\boldsymbol{a}, \mathbf{m}$ & $\boldsymbol{\alpha}, \mathbf{r a d}$ & $\boldsymbol{f}(\boldsymbol{i})$ & $\boldsymbol{n} \boldsymbol{s}(\boldsymbol{i})$ \\
\hline 6.2 & $\pi / 2$ & 0 & -0.349 & $\pi$ & 6 & 2 \\
6.3 & $\pi / 2$ & 0 & -0.349 & 0 & 6 & 2 \\
7.2 & $\pi / 2$ & 0 & 0.2 & 0 & 7 & 2 \\
\hline
\end{tabular}


By the tree-like graph (Fig. 2) presenting the robotic kinematic structure, we define the reachability matrix $D(21 \times 21)$ of the robot's actuating links. Its elements' numerical values can be found in Table 3 .

Table 3. Numerical values of the matrix $\boldsymbol{D}$ elements

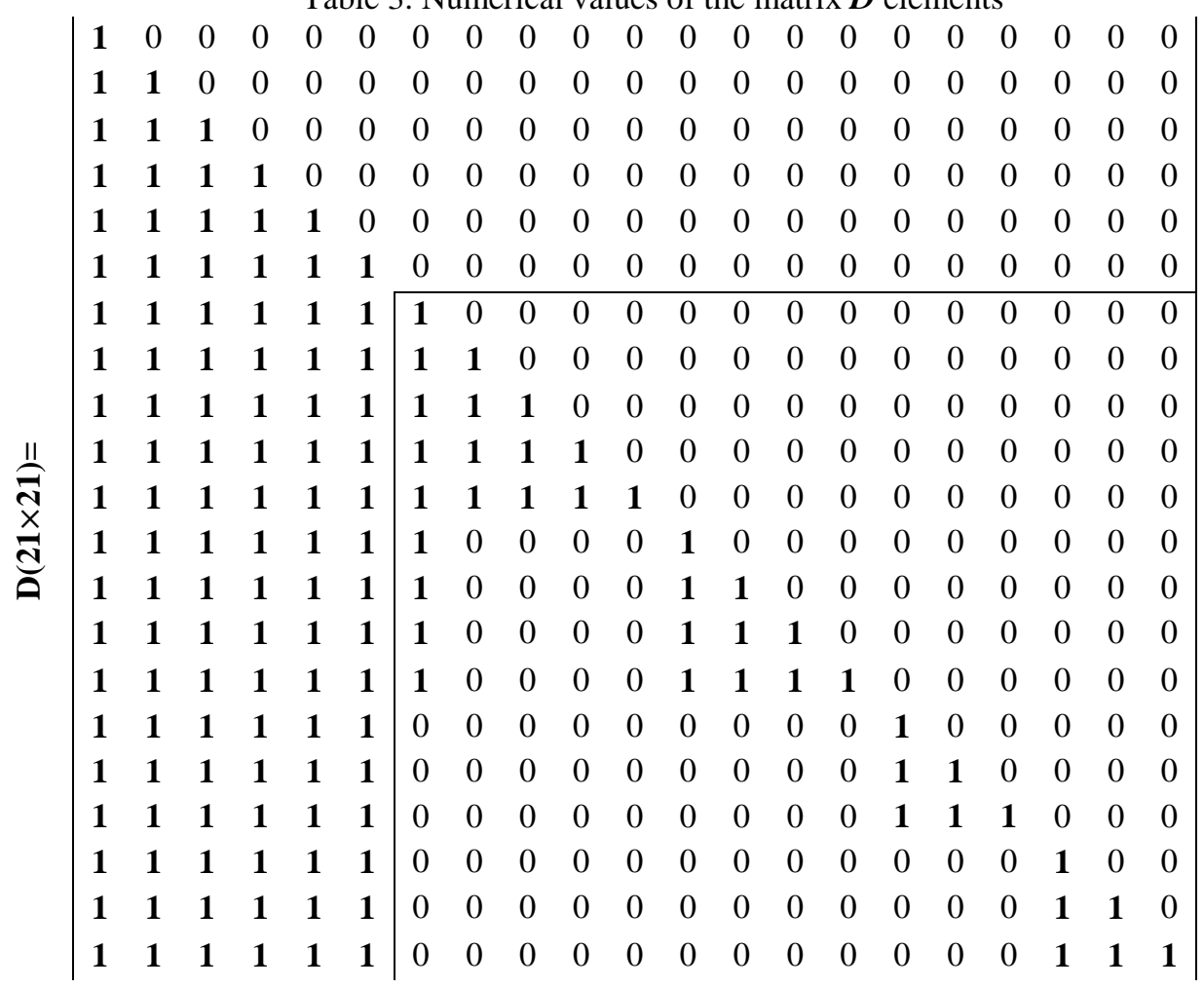

The first six columns of the matrix $D(21 \times 21)$ correspond to the links of the dummy kinematic chain connecting the robot's body with a dummy stand stationary in the absolute system. The 1-value elements of the first six columns indicate that from these links are reachable all the other links of the robot's kinematic chain. The seventh column of the matrix $D(21 \times 21)$ corresponds to Link 7 , from which the links $8 \div 15$ are reachable. The eighth corresponds to Link 8 , from which only the links $9 \div 11$ are reachable. Link 11 (the $11^{\text {th }}$ column) is reachable only from itself, etc.

Thus, if we complement the traditional DH parameters $\theta_{i}, d_{i}, a_{i}, \alpha_{i}$ with the parameters $f(i), n s(i)$, as well as the block vector $\bar{z}$, the diagonal matrix $\sigma$ and the reachability matrix $D$ characterizing the specific CS's features, it is possible to formalize the kinematic equations for the actuating mechanism of a robot with an arbitrary tree-like kinematic chain.

Using the above-proposed method of describing the kinematics of a tree-like robot's AM with the help of the modified DH coordinate system, we write kinematic dependencies for all AM's links in a block matrix form $[13,14]$. The values' symbols used in the below expressions $(5) \div(14)$ correspond to those in Kovalchuk et al. [14]. The expressions to define the links' angular velocities ${ }^{0} W$ and angular accelerations ${ }^{0} e$ can be formed as follows:

$$
\begin{gathered}
{ }^{0} W=\left(D \cdot{ }^{0} z^{d} \cdot \sigma\right) \dot{q} ; \\
{ }^{0} e=\left(D \cdot{ }^{0} z^{d} \cdot \sigma \cdot \ddot{q}+D \cdot \sigma \cdot \dot{q}^{d} \cdot{ }^{0} \dot{z}\right) .
\end{gathered}
$$

Let us write the expressions defining the velocities ${ }^{0} V$ and accelerations ${ }^{0} \dot{V}$ of the links' primary CS origins:

$$
\begin{array}{r}
{ }^{0} V=\left(\Lambda^{T}\left({ }^{0} \mathrm{l}_{D}\right){ }^{0} Z^{d} \cdot \sigma+D \cdot{ }^{0} Z^{d} \cdot(E-\sigma)\right) \dot{q} \\
{ }^{0} \dot{V}=\left(\Lambda^{T}\left({ }^{0} \mathrm{l}_{D}\right){ }^{0} z^{d} \cdot \sigma+D \cdot{ }^{0} z^{d} \cdot(E-\sigma)\right) \ddot{q}+
\end{array}
$$




$$
+\left(\Lambda^{T}\left({ }^{0} \dot{\mathrm{l}}_{D}\right){ }^{0} \dot{Z}^{d} \cdot \sigma+\Lambda^{T}\left({ }^{0} \dot{\mathrm{l}}_{D}\right){ }^{0} z^{d} \cdot \sigma+D \cdot{ }^{0} \dot{Z}^{d} \cdot(E-\sigma)\right) \dot{q}
$$

The AM links' center of mass acceleration can be determined with the following expression:

$$
\begin{aligned}
& { }^{0} \bar{a}_{\text {L.M. }}=\left(D \cdot{ }^{0} z^{d} \cdot(E-\sigma)+\Lambda^{T}\left({ }^{0} C_{f D}\right){ }^{0} z^{d} \cdot \sigma\right) \ddot{q}+ \\
& +\Lambda^{T}\left({ }^{0} C_{f D}\right) \Lambda^{T}\left({ }^{0} z^{d} \cdot \sigma \cdot \dot{q}^{d}\right)(D-E){ }^{0} z^{d} \cdot \sigma \cdot \dot{q}+ \\
& +\Lambda^{T}\left(\Lambda^{T}\left({ }^{0} C_{f D}\right) \cdot \sigma \cdot{ }^{0} \dot{q}^{d} \cdot{ }^{0} \dot{z}^{d}+D+\Lambda^{T}\left({ }^{0} C_{f D}\right) \cdot\right. \\
& \left.\quad \cdot\left((D-E) \cdot \sigma \cdot{ }^{0} z^{d} \cdot \dot{q}^{d}\right)\right){ }^{0} z^{d} \cdot \sigma \cdot \dot{q}+ \\
& \quad+2 \cdot D \cdot \Lambda^{T}\left({ }^{0} Z^{d}\right) \cdot(E-\sigma) \cdot \dot{q}^{d}(D-E){ }^{0} z^{d} \cdot \sigma \cdot \dot{q} .
\end{aligned}
$$

Each expression $(5 \div 9)$ contains the above defined reachability matrix $D$, block vector $z$ (it describes the sequence of the basis vectors of the links-related coordinate systems' $z$ ) and diagonal matrix $\sigma$ (it determines the joints' types for AM links - translational or rotational). The superscript " 0 " indicates that all the obtained expressions $(5 \div 9)$ are written in the reference CS " 0 ".

The above-proposed method also allows writing dynamic relations for all robotic actuating links in a block-matrix form. The diagram showing the application of forces and moments existing between the treelike AM's links is represented in Figure 4.

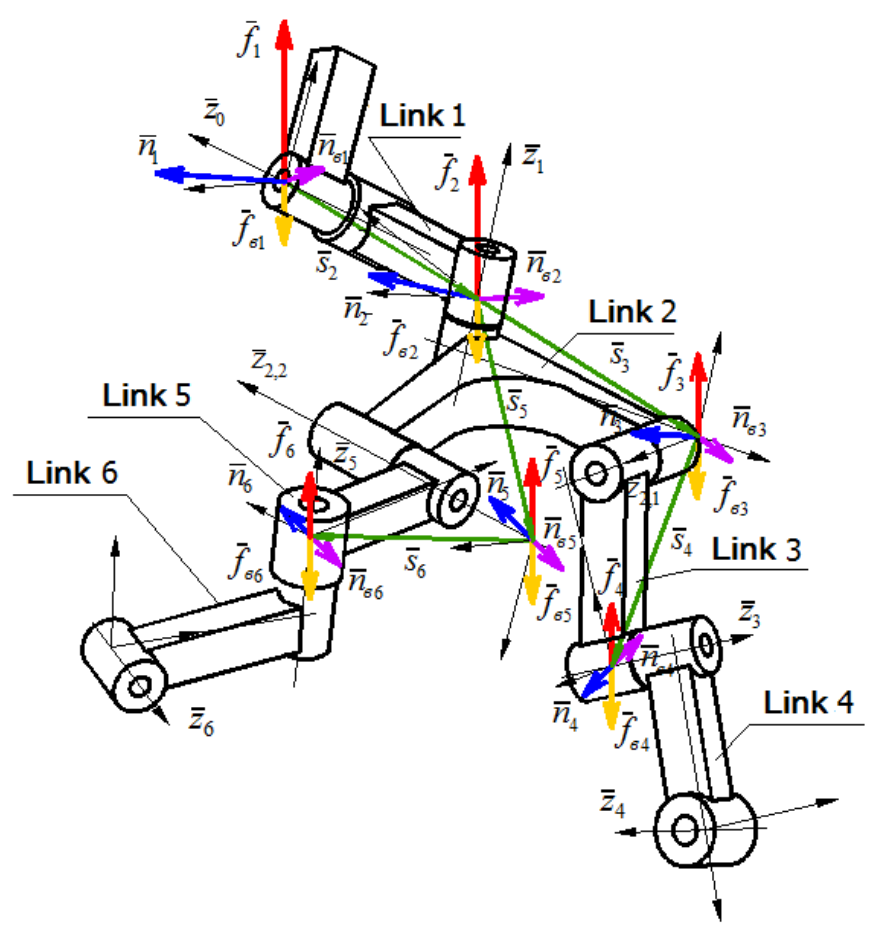

Figure 4. Forces and moments existing between the robotic tree-like actuating mechanism's links

14]:

We can write the expressions describing dynamic relations if the following conditions are true [13,

- the forces and moments existing between the robot's AM links are to be considered through the forces and moments affecting the link from its ancestor's side;

- the forces acting on the links from their ancestors, as well as the link-affecting external forces are brought to the origins of the coordinate systems of the ancestors corresponding to these links;

- the moments acting on the links include the moments obtained after bringing these forces to the origins of corresponding coordinate systems. 
Then the block-matrix expressions defining the forces ${ }^{0} f$ and the moments ${ }^{0} n$ existing between the AM's links can be written as follows:

$$
\begin{gathered}
{ }^{0} f=D^{T} \cdot m^{d} \cdot{ }^{0} a \cdot D^{T} \cdot{ }^{0} f_{\mathrm{B}} \\
{ }^{0} n=-\left[\Lambda^{T}\left({ }^{0} C_{f D}\right)\right]^{T} \cdot m^{d} \cdot{ }^{0} a+D^{T} \cdot{ }^{0} J_{C}^{d} \cdot{ }^{0} e+D^{T} \cdot \Lambda\left({ }^{0} W^{d}\right) \cdot{ }^{0} J_{C}^{d} \cdot \\
\cdot{ }^{0} W-\left(D^{T}-E\right) \cdot \Lambda\left({ }^{0} s^{d}\right) \cdot D^{T} \cdot{ }^{0} f_{\mathrm{B}}-D^{T} \cdot{ }^{0} n_{\mathrm{B}}-D^{T} \cdot \Lambda\left({ }^{0} t^{d}\right) \cdot{ }^{0} f_{\mathrm{B}} ;
\end{gathered}
$$

The expressions for determining the forces and moments developed by robotic actuators can be obtained by projecting the above-defined forces ${ }^{0} f$ and moments ${ }^{0} n$ of the links' interaction onto the axes $z$ of the coordinate systems, which correspond to the joints connecting these links with their ancestors.

$$
\begin{gathered}
\tau=\sigma \cdot\left({ }^{0} z^{d}\right)^{T} \cdot\left[-\left(\Lambda\left({ }^{0} C_{f D}\right)\right)^{T} \cdot m^{d} \cdot{ }^{0} a+D^{T} \cdot{ }^{0} J_{C}^{d} \cdot{ }^{0} e+\right. \\
+D^{T} \cdot \Lambda\left({ }^{0} W^{d}\right) \cdot{ }^{0} J_{C}^{d} \cdot{ }^{0} W-\left(\left(D^{T}-E\right) \cdot \Lambda\left({ }^{0} s^{d}\right) \cdot D^{T}+\right. \\
\left.\quad+D^{T} \cdot \Lambda\left({ }^{0} t^{d}\right) \cdot{ }^{0} f_{\mathrm{B}}-D^{T} \cdot{ }^{0} n_{\mathrm{B}}\right]+ \\
+(E-\sigma) \cdot\left({ }^{0} z^{d}\right)^{T} \cdot\left(D^{T} \cdot m^{d} \cdot{ }^{0} a-D^{T} \cdot{ }^{0} f_{\mathrm{B}}\right) .
\end{gathered}
$$

If we describe, for the links' forces in the expression (12), velocities and accelerations through the generalized coordinates $\boldsymbol{q}$ and their derivatives and group the summands at absolute velocities and accelerations, we get the following dynamic equation for the actuating mechanism of a robot with the treelike kinematic structure:

where:

$$
A(q) \cdot \ddot{q}+B(q, \dot{q})-C(q) \cdot{ }^{0} f_{\mathrm{B}}-H(q) \cdot{ }^{0} n_{\mathrm{B}}=\tau,
$$

$$
\begin{aligned}
& A(q)=\sigma \cdot\left({ }^{0} z^{d}\right)^{T} \cdot\left(-\left(\Lambda\left({ }^{0} C_{f D}\right)\right)^{T} \cdot m^{d} \cdot\left(D \cdot{ }^{0} z^{d} \cdot(E-\sigma)+\Lambda^{T}\left({ }^{0} C_{f D}\right) \cdot\right.\right. \\
& \left.\cdot{ }^{0} Z^{d} \cdot \sigma\right)+D^{T} \cdot{ }^{0} J_{C}^{d} \cdot D \cdot{ }^{0} Z^{d} . \\
& \cdot \sigma)+(E-\sigma) \cdot\left({ }^{0} Z^{d}\right)^{T} \cdot D^{T} \cdot m^{d} \cdot\left(D \cdot{ }^{0} Z^{d} \cdot(E-\sigma)+\Lambda^{T}\left({ }^{0} C_{f D}\right) \cdot{ }^{0} Z^{d} \cdot \sigma\right) \text {; } \\
& B(q, \dot{q})=\sigma \cdot\left({ }^{0} Z^{d}\right)^{T} \cdot\left\{-\left(\Lambda\left({ }^{0} C_{f D}\right)\right)^{T} \cdot m^{d} \cdot\left[\Lambda^{T}\left({ }^{0} C_{f D}\right) \cdots \Lambda^{T}\left({ }^{0} Z^{d} \cdot \sigma \cdot \dot{q}^{d}\right) \cdot(D-E)+\right.\right. \\
& +\Lambda^{T}\left(\Lambda^{T}\left({ }^{0} C_{f D}\right) \cdot \sigma \cdot \dot{q}^{d} \cdot{ }^{0} Z^{d} \cdot D+\Lambda^{T}\left({ }^{0} C_{f D}\right) \cdot\left((D-E) \cdot \sigma \cdot{ }^{0} Z^{d} \cdot \dot{q}\right)^{d}\right)+ \\
& \left.+2 \cdot D \cdot \Lambda^{T}\left({ }^{0} Z^{d} \cdot(E-\sigma) \cdot \dot{q}^{d}\right) \cdot(D-E)\right]+D^{T} \cdot{ }^{0} J_{C}^{d} \cdot D \cdot \sigma \cdot \dot{q}^{d} \cdot \Lambda^{T} \cdot .\left({ }^{0} Z^{d}\right) \cdot(D-E)+ \\
& \left.+D^{T} \cdot \Lambda\left(D \cdot{ }^{0} Z^{d} \cdot \sigma \cdot \dot{q}\right)^{d} \cdot{ }^{0} J_{C}^{d} \cdot D\right\} \cdot{ }^{0} Z^{d} \cdot \sigma \cdot \dot{q}+(E-\sigma) \cdot \\
& \cdot\left({ }^{0} Z^{d}\right)^{T} \cdot D^{T} \cdot m^{d} \cdot\left[\Lambda^{T}\left({ }^{0} C_{f D}\right) \cdot \Lambda^{T}\left({ }^{0} Z^{d} \cdot \sigma \cdot \dot{q}^{d}\right) \cdot\right. \\
& \cdot(D-E)+2 \cdot D \cdot \Lambda^{T}\left({ }^{0} Z^{d} \cdot(E-\sigma) \cdot \dot{q}^{d}\right) \cdot(D-E)+ \\
& +\Lambda^{T}\left(\Lambda^{T}\left({ }^{0} C_{f D}\right) \cdot \sigma \cdot \dot{q}^{d} \cdot{ }^{0} Z^{d} \cdot D+\right. \\
& +\Lambda^{T}\left(\Lambda^{T}\left({ }^{0} C_{f D}\right) \cdot \sigma \cdot \dot{q}^{d} \cdot{ }^{0} Z^{d} \cdot D+\Lambda^{T}\left({ }^{0} C_{f D}\right) \cdot\right. \\
& \left.\left.\cdot\left((D-E) \cdot \sigma \cdot{ }^{0} Z^{d} \cdot \dot{q}\right)^{d}\right)\right]^{0} \cdot{ }^{0} Z^{d} \cdot \sigma \cdot \dot{q} ; \\
& C(q)=\sigma \cdot\left({ }^{0} z^{d}\right)^{T} \cdot\left(\left(D^{T}-E\right) \cdot \Lambda\left({ }^{0} s^{d}\right) \cdot D^{T}+D^{T} \cdot \Lambda\left({ }^{0} t^{d}\right)\right)+ \\
& +(E-\sigma) \cdot\left({ }^{0} Z^{d}\right)^{T} \cdot D^{T} \text {; } \\
& H(q)=\sigma \cdot\left({ }^{0} z^{d}\right)^{T} \cdot D^{T} \text {; } \\
& { }^{0} \bar{f}_{6}=\left({ }^{0} \bar{f}_{b 1},{ }^{0} \bar{f}_{62}, \ldots{ }^{0} \bar{f}_{6 N}\right)^{T} \text { и }{ }^{0} \bar{n}_{b}=\left({ }^{0} \bar{n}_{b 1},{ }^{0} \bar{n}_{b 2}, \ldots{ }^{0} \bar{n}_{b N}\right)
\end{aligned}
$$

The block matrices of external forces and moments acting on the robotic tree-like actuating mechanism's links.

This equation is valid under the following assumptions:

- the actuating mechanism's links absolutely rigid;

- the joints' connections are holonomic;

- the kinematic chains of the tree-like kinematic structure are open.

The resulting equation (13) based on the modified DH coordinate system is universal (under the

Denavit-Hartenberg Coordinate System for Robots with Tree-like Kinematic Structure (Aleksander K) 
indicated assumptions) for a whole class of actuating mechanisms of the robots with tree-like kinematic structures defining by reachability matrices $D$, block vectors $\bar{Z}$ and diagonal matrices $\sigma$.

\section{DISCUSSION}

A. K. Kovalchuk [15] presents the results of studying the kinematics and dynamics of a robotic dog's tree-like actuating mechanism. For the AM with 22 degrees of motion freedom, the author obtained the numerical values of the modified DH parameters, the reachability matrix $D$, the block vector $\bar{z}$ and the diagonal matrix $\sigma$. Based on the equation (13) and using a specially developed MATLAB software [16], there are defined moment and capacity values in the freedom degrees of the robot dog.

The work of A. K. Kovalchuk [17] is devoted to KS synthesis and studying the dynamic of a robotic crab tree-like actuating mechanism with 62 degrees of motion freedom. The modified DH coordinate system can be applied in building a mathematical model of its actuating mechanism's kinematics and dynamics. The developed 3-D model of a robotic crab AM helped to find the mass and inertia characteristics of its construction elements. By means of the software of Kovalchuk et al. [16], the author obtained the numeric values of the matrices' elements included into the equation (13), as well as the values of moments and capacities in the freedom degrees of the robotic crab.

A. K. Kovalchuk [18] considers the example of using the modified DH CS in forming the mathematical model of a tree-like AM's kinematics and dynamics for an anthropomorphic robot having 114 degrees of motion. For the equation (13), scientists received the values of the elements of its constituent matrices $A(q), B(q, \dot{q}), C(q), H(q)$ and calculated, with the help of the software (Kovalchuk et al., 2012), the moment and capacity values in the motion freedom degrees of the robot's AM. The obtained results are recommended for use in creating modern pieces of anthropomorphic walking robots.

In the works of Kovalchuk [19,20], it is shown that the modified DH CS is an effective means to form mathematical models of a robotic AM with a linear open kinematic chain, which is a particular case of a tree-like $\mathrm{KC}$.

In this case, the kinematic scheme may be represented as an oriented graph that has no cycles. The graph's vertices are connected in series, because each of them has no more than two adjacent vertices.

The reachability matrix $D$ of the robot's AM links is the degenerated into a lower triangular unit matrix of the order $N$, where $N$ is the number of freedom degrees for the robot's AM.

If the gripping manipulator or any other AM's link is imposed with external connections, the AM dynamic equation is written in the following form [14]:

$$
\left(\begin{array}{cc}
A(q) & -J_{V R}^{T}(q) \\
J_{t}(q) & 0
\end{array}\right) \cdot\left(\begin{array}{c}
\ddot{q} \\
{ }^{0} R^{f}
\end{array}\right)+\left(\begin{array}{c}
B(q, \dot{q}) \\
P(q)
\end{array}\right)-\left(\begin{array}{c}
L(q) \cdot{ }^{0} F_{\beta} \\
0
\end{array}\right)=\left(\begin{array}{l}
\tau \\
0
\end{array}\right)
$$

The matrix coefficients $A(q), B(q, \dot{q}), J_{t}(q), J_{V R}^{T}(q),{ }^{0} R^{f}, P(q), L(q),{ }^{0} F_{B}$ are determined in accordance with [14].

The equation (14) can determine the motion of the robotic actuating mechanism with an arbitrary tree-like KS with imposed drive connections and calculate the values of the connections' appearing reaction forces and moments.

A. A. Vereykin et al. [21] show the effectiveness of the proposed coordinate system for constructing equations of kinematics and dynamics for the tree-like actuating mechanism of an active exoskeleton. Using the equation (14) made it possible not only to determine the driving power values in motion degrees of its AM with imposed drive connections but also to calculate the reaction forces and moments of these connections, which appear upon interacting with the base surface for steps. The specially developed MATLAB-based program [22] was an effective means of the performed calculations and research.

The study of Kovalchuk et al. [23] describes the method of KS synthesis for a walking robot's treelike actuating mechanisms - from the photographic images of its biological prototype's skeleton. The method is based on a KS reconstruction algorithm [24-26] and the modified DH CS. There is an example illustrating the results of the method's application - creating the kinematic structure of an actuating mechanism for a robot-Stegosaurus.

Thus, the above-mentioned research results can lead to the conclusion that the proposed modified Denavit-Hartenberg coordinate system has a common practical ground and can be used in forming mathematical models of kinematics and dynamics for the actuating mechanisms of robot with an arbitrary kinematic structure.

\section{CONCLUSIONS}

IJRA Vol. 5, No. 4, December 2016: $244-254$ 
Creating modern walking robots is constrained by the lack of effective methods of forming the mathematical models of their actuating mechanisms with tree-like kinematic structures.

In constructing such mechanisms' mathematical models of kinematics and dynamics, it is proposed to use the modified Denavit-Hartenberg coordinate system. Its application provides a developer даёт with a clear algorithm of building mathematical models of robotic actuating mechanisms with arbitrary tree-like kinematic structure.

The mathematical models obtained by this method and the software developed for such research can be applied in creating advanced sample walking robots.

\section{REFERENCES}

[1] V. S. Medvedev, A. G. Leskov, and A. S. Yushchenko, Control Systems of Robotic Manipulators. E. P. Popov, ed. Moscow, Russia: Nauka, 1978 (in Russian).

[2] K. V.Frolov and E. I.Vorobyov, eds., The Mechanics of Industrial Robots. Moscow, Russia: Vysshaya Shkola, 1988 (in Russian).

[3] K. Fu, R. Gonzalez, and C. Lee, Robotics: Control, Sensing, Vision and Intelligence. New York: McGraw-Hill, 1987.

[4] M. Shahinpoor, A Robot Engineering Textbook. New York: Harper and Row, 1987.

[5] P. D. Krut'ko, Robotic Executive Systems Control. Moscow, Russia: Nauka, 1991 (in Russian).

[6] S. L. Zenkevich and A. S. Yushchenko, Fundamentals of Manipulation Robots Control. Moscow, Russia: Bauman Technical Uniersity, 2004 (in Russian).

[7] A. G. Leskov and A. S. Yushchenko, Modeling and Analysis of Robotic Systems. Moscow, Russia: Mashinostroyeniye, 1992 (in Russian).

[8] A. G. Leskov, Theoretical Foundations to Modeling and Analysis of Manipulation Robots Dynamics, Their Application in Problems of Designing and Preparing Operators. Habilitation Thesis. Moscow, Russia: Bauman Technical Uniersity, 2002 (in Russian).

[9] A. G. Leskov, K. V. Bazhinova, S. D. Moroshkin, and E. V. Feoktistova, "Building Kinematic Models of Robotic Manipulators' Actuating Mechanisms Using Block Matrices," Inzhenernyi Zhurnal: Nauka i Innovatsii, no. 9, 2013. Available from: http://engjournal.ru/catalog/pribor/robot/954.html (in Russian).

[10] J. Denavit and R. S. Hartenberg, "Kinematic notation for lower-pair mechanisms based on matrices," J. Appl. Mech, vol. 77, pp. 215-221, 1955.

[11] A. K. Kovalchuk, "Development of a Mathematical Model of the Actuating Mechanism of Robotic Mannequin," Nauchnyi Vestnik Moskovskogo Gosudarstvennogo Tekhnicheskogo Universiteta Grazhdanskoy Aviatsii, vol. 168, no. 6, pp. 103-109, 2011 (in Russian).

[12] V. E. Alekseev and V. A. Talanov, Graphs and Algorithms. Data Structures. Computational Models. Moscow, Russia: Binom, 2006 (in Russian)

[13] A. K. Kovalchuk, D. B. Kulakov, and S. E. Semenov, "Mathematical Description of Kinematics and Dynamics for Robotic Actuators with a Tree-like Kinematic Structure," Izvestiya Vysshikh Uchebnykh Zavedenii. Seriya Mashinostroyeniye, vol. 11, pp. 13-25, 2008 (in Russian).

[14] A. K. Kovalchuk, B. B. Kulakov, D. B. Kulakov, S. E. Semenov, and V. V. Yarots. Basic Theory of Walking Robots' Actuating Mechanisms. Moscow, Russia: Rudomino, 2010 (in Russian).

[15] A. K. Kovalchuk, "The Selection of a Kinematic Structure and the Study of the Tree-like Actuating Mechanism of a Robot Dog," Izvestiya Vysshikh Uchebnykh Zavedenii. Seriya Mashinostroyeniye, vol. 8, pp. 65-73, 2011 (in Russian).

[16] A. K. Kovalchuk, L. A. Karginov, B. B. Kulakov, D. B. Kulakov, S. E. Semenov, and V. V. Yarots, "The Program to Simulate Tree-like Actuating Mechanisms of Walking Robots," Certificate of state registration of software, no. $2012610398,10.01 .2012$.

[17] A. K. Kovalchuk, "The Selection of a Kinematic Structure and the Study of the Tree-like Actuating Mechanism Dynamics of a Robot Crab," Izvestiya Vysshikh Uchebnykh Zavedenii. Seriya Mashinostroyeniye, vol. 7, pp. 73-79, 2013 (in Russian).

[18] A. K. Kovalchuk, "Designing of an Actuating Mechanism for an Anthropomorphic Walking Robot," Yestestvenniye i Tekhnicheskiye Nauki, vol. 2, no. 70, pp. 162-166, 2014 (in Russian).

[19] A. K. Kovalchuk, "Calculation of a Robot Driving Power Considering Its Actuating Mechanism Dynamics," Yestestvenniye i Tekhnicheskiye Nauki, vol. 1, no. 69, pp. 128-131, 2014 (in Russian).

[20] A. K. Kovalchuk, "Designing Drives of a Medical Robot Actuator," Life Science Journal, no. 11s, pp. 337-340, 2014.

[21] A. A.Vereykin, A. K. Kovalchuk, and L. A. Karginov, "A Study of The Dynamics of a Lower Limb Exoskeleton Actuating Mechanism, Taking into Account the Reactions of the Bearing Surface," Nauka i Obrazovaniye. Electronic Journal of Bauman Technical University, Moscow, Russia, vol. 12, pp. 256-278, 2014. DOI: 10.7463 / 0815.9328000 (in Russian).

[22] A. K. Kovalchuk, L. A. Karginov, B. B. Kulakov, D. B. Kulakov, S. E. Semenov, V. V. Yarots, and A. A. Vereykin, "Simulation of Tree-like Walking Robot Actuating Mechanisms, Taking into Account Imposed External Connections. Certificate of state registration of software, no. 2014612547, 28.02.2014.

[23] A. K. Kovalchuk, L. A. Karginov, F. Kh. Akhmetova, A. Y. Ustyuzhanin, S. S. Sekerin, and A. A. Vereykin, "The Synthesis of a Kinematic Scheme for the Tree-like Actuating Mechanism of a Robotic Stegosaurus Using the

Denavit-Hartenberg Coordinate System for Robots with Tree-like Kinematic Structure (Aleksander K) 
Photographic Images of Its Biological Prototype,” Nauka i Obrazovaniye. Electronic Journal of Bauman Technical University, Moscow, Russia, no. 05, pp. 82-102, 2015. DOI: 10.7463 / 0515.0766349 (in Russian).

[24] K. A. Pupkov, A. K. Kovalchuk, and B. B. Kulakov, "Use of Biological Prototypes in Constructing the Kinematic Schemes of Modern Walking Robots," Vestnik RUDN. Inzhenerniye Issledovaniya, vol. 4, pp. 44-54, 2009 (in Russian).

[25] K. A. Pupkov, A. K. Kovalchuk, and B. B. Kulakov, "Usage of Biological Prototypes for Kinematical Scheme Construction of Modern Robots," in: Preprints of the 13th IFAC Symposium on Information Control Problems in Manufacturing, 3-5 June 2009, pp. 1829-1834. Moscow (in Russian).

[26] A. K. Kovalchuk, "Use of Biological Prototypes in Designing Tree-like Actuating Mechanisms of Bipedal Walking Robots," Izvestiya Vysshikh Uchebnykh Zavedenii. Seriya Mashinostroyeniye, vol. 9, pp. 49-56, 2011 (in Russian).

\section{BIOGRAPIES OF AUTHORS}

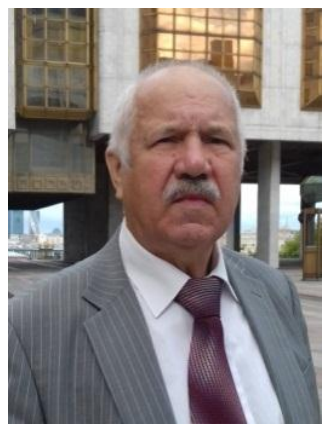

Aleksander K. Kovalchuk, Ph.D. (Engineering), Associate Professor in Bauman Moscow State University, author of more than 100 research publications, monographs and textbooks for university students. His research interests are control algorithms and walking robot actuator systems. Main recent publications: A. K. Kovalchuk (ed.). Designing actuator systems for walking robots with tree-like kinematic structure [Textbook]. Moscow, Bauman Moscow State Technical University, 2013, 115 p. (in Russian). S. D. Kugultinov, A. K. Kovalchuk, I. I. Portnov. Engineering materials processing technologies [Textbook]. 3rd edn., revised and enlarged. Moscow, Bauman Moscow State Technical University, 2010, 678 p. (in Russian). A. K. Kovalchuk, S. V. Shaitura. Geographic information systems principles [Textbook]. Moscow, Rudomino, 2009, 209 p. (in Russian). S. D. Kugultinov, A. K. Kovalchuk, I. I. Portnov. Cutting rocket engineering materials [Textbook]. Moscow, Bauman Moscow State Technical University, 2008, 192 p. (in Russian).

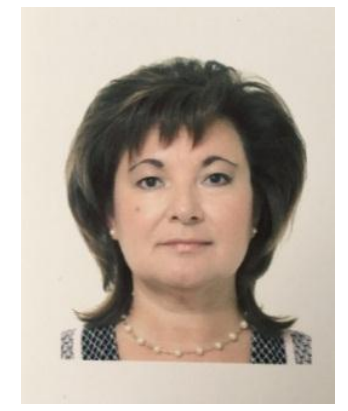

Faniya Kh. Akhmetova, Ph.D. (Physics and Mathematics), Associate Professor in Bauman Moscow State Technical University, author and co-author of more than 40 research and methodological publications. Her research interests include applied mathematics and mathematical simulation. Main recent publications: F. Kh. Akhmetova. Principles of analysis. Theory of limits. Moscow, Bauman Moscow State Technical University, 2014 (in Russian). F. Kh. Akhmetova, P. A. Vlasov. MathCAD. Mathematical analysis problem solving: Integrating. Mocsow, Bauman Moscow State Technical University, 2008, 34 p. (in Russian). F. Kh. Akhmetova, B. T. Dobritsa, A. V. Syrtsov. Indefinite integration [Methodology recommendations for teachers]. Moscow, Bauman Moscow State Technical University, 2008, 48 p. (in Russian). F. Kh. Akhmetova et al. Footballer robots: The issues of design and control. Moscow, Bauman Moscow State Technical University, 2007, 108 p. (in Russian). 